\title{
Robot-Assisted Cardiac Surgery
}

\author{
Norihiko Ishikawa, MD, $\mathrm{PhD}$ and Go Watanabe, $\mathrm{MD}, \mathrm{PhD}$
}

\begin{abstract}
Recognition of the significant advantages of minimizing surgical trauma has resulted in the development of minimally invasive surgical procedures. Endoscopic surgery offers patients the benefits of minimally invasive surgery, and surgical robots have enhanced the ability and precision of surgeons. Consequently, technological advances have facilitated totally endoscopic robotic cardiac surgery, which has allowed surgeons to operate endoscopically rather than through a median sternotomy during cardiac surgery. Thus, repairs for structural heart conditions, including mitral valve plasty, atrial septal defect closure, multivessel minimally invasive direct coronary artery bypass grafting (MIDCAB), and totally endoscopic coronary artery bypass graft surgery (CABG), can be totally endoscopic. Robot-assisted cardiac surgery as minimally invasive cardiac surgery is reviewed.
\end{abstract}

Keywords: robotic surgery, minimally invasive surgery, CABG, mitral valve plasty, ASD closure

The significant advantages of minimizing surgical trauma, such as reduced pain, shorter hospital stays, faster return to normal activities, and improved cosmesis, have resulted in the development of minimally invasive surgery. ${ }^{1)}$ Until recently, various difficulties associated with endoscopic approaches had stalled similar progress in the field of cardiac surgery. However, robotic technology overcame the difficulties associated with conventional endoscopic surgery and has made possible a new approach to minimally invasive cardiac surgery.

The application of robot-assisted coronary surgery ranges from internal mammary artery (IMA) harvesting with handsewn anastomoses to totally endoscopic coronary artery bypass grafting (TECAB) either on- or off-pump. The bilateral IMAs can be harvested with the aid of a surgical robot, and then multivessel bypass grafting can follow. Srivastava calls such robot-assisted minimally invasive direct coronary

NewHeart Watanabe Institute, Tokyo, Japan

Received: May 15, 2015; Accepted: June 8, 2015

Corresponding author: Norihiko Ishikawa, MD, PhD. NewHeart Watanabe Institute, 3-19-11 Hamadayama, Suginami-ku, Tokyo 168-0065, Japan

Email: iskwnrhk@gmail.com

(C)2015 The Editorial Committee of Annals of Thoracic and Cardiovascular Surgery. All rights reserved. artery bypass grafting (MIDCAB) "ThoraCAB." 2 ) Surgical robots can not only endoscopically harvest the IMA, but they can also anastomose the coronary artery in TECAB.

On the other hand, closed-chest cardiopulmonary bypass (CPB) and cardioplegic arrest have stimulated the development of minimally invasive cardiac surgery (MICS) via a small thoracotomy, ${ }^{3)}$ and this has now become universal, especially to treat valve diseases and congenital structural heart diseases. Endoscopic techniques are also required for MICS, but conventional endoscopic instrumentation lacks the dexterity required for delicate cardiac surgical procedures, and the loss of depth perception caused by twodimensional monitors further increases operative obstacles.

Surgical robots have been developed to enhance surgical ability and precision, and to repair structural heart conditions, including mitral valve plasty (MVP), atrial septal defect closure, cardiac tumour resection, ThoraCAB, and TECAB. The most common applications in cardiac surgery are MVP and endoscopic coronary artery bypass grafting (CABG).

\section{da Vinci Surgical System}

The da Vinci surgical system (Intuitive Surgical Inc., Sunnyvale, CA, USA) comprises a surgeon's console, a 

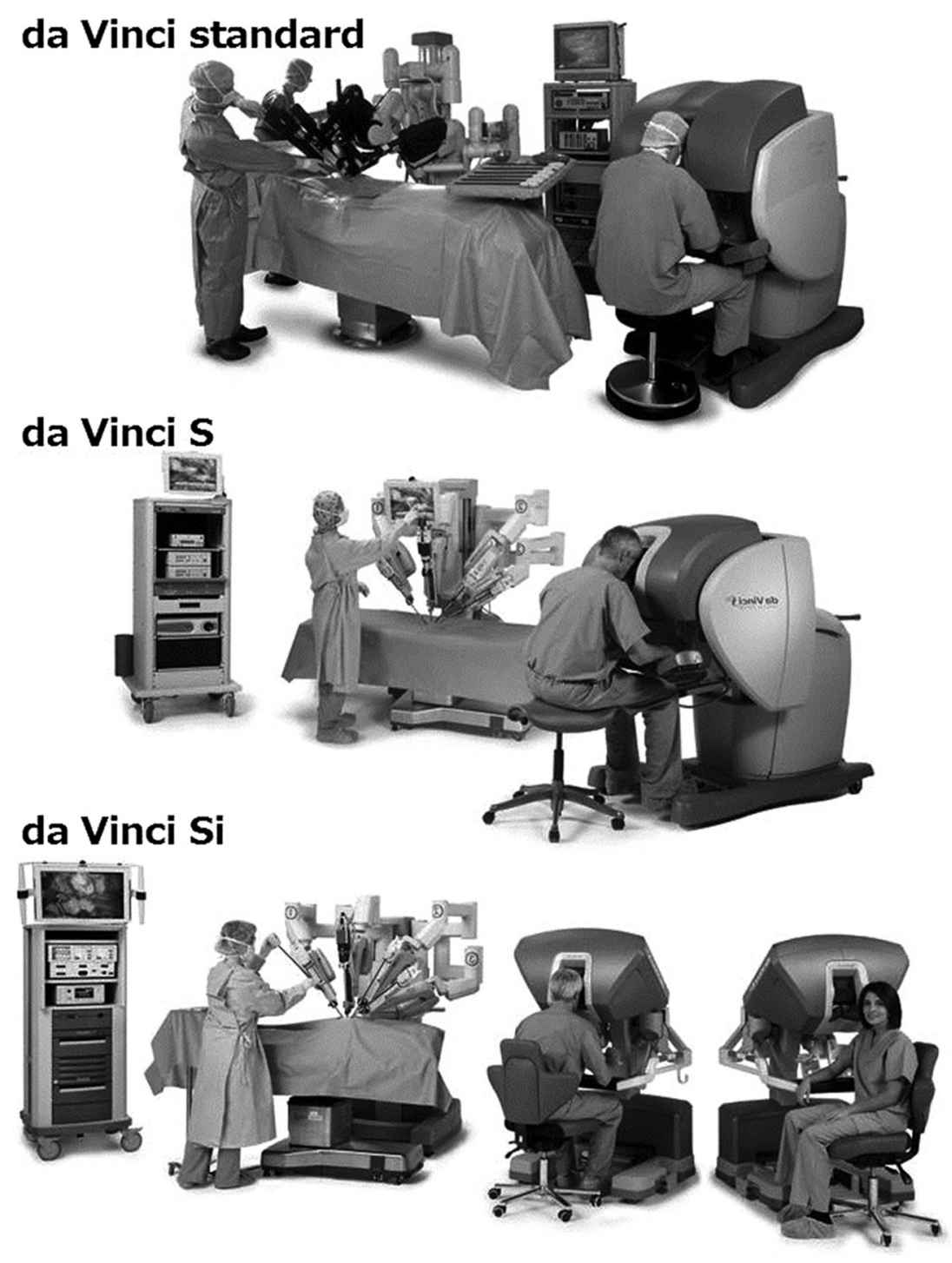

Fig. 1 The da Vinci surgical system.

surgical cart, and a vision cart (Fig. 1). The surgeon at the console manipulates two master handles at the master remote console and is able to acquire high-resolution, binocular, three-dimensional, magnified views of operative fields equivalent to those of open surgery. The system can be downscaled by adjusting the ratio of the motions of the handles to that of the surgical instruments, and a motion filter prevents unintended movements caused by human tremor. These technical advantages permit high-precision microsutures in a deep operative field through a small incision. The Ministry of Health, Labour and Welfare of Japan approved the second and third da Vinci surgical systems as medical devices for robotic thoracoscopic and laparoscopic procedures in 2009 and 2012, respectively, but not for cardiac surgical procedures.

\section{Robotic Surgery for Ischemic Heart Disease}

\section{ThoraCAB}

The short-term patency rates of off-pump coronary artery bypass grafting (OPCAB) are similar to those of conventional coronary artery bypass grafting $(\mathrm{CABG}){ }^{4,5)}$ In addition, compared with $\mathrm{CABG}, \mathrm{OPCAB}$ is associated with a reduced postoperative length of stay, lesser blood and blood component usage, and an earlier return to normal lifestyle. $\left.{ }^{6}\right)$ MIDCAB has all the benefits of $\mathrm{OPCAB}$, and this procedure avoids the morbidity caused by median sternotomy.

Randomized, controlled trials to evaluate pain, time to return to a normal lifestyle, and graft patency should be conducted to validate the long-term efficacy of MIDCAB. 


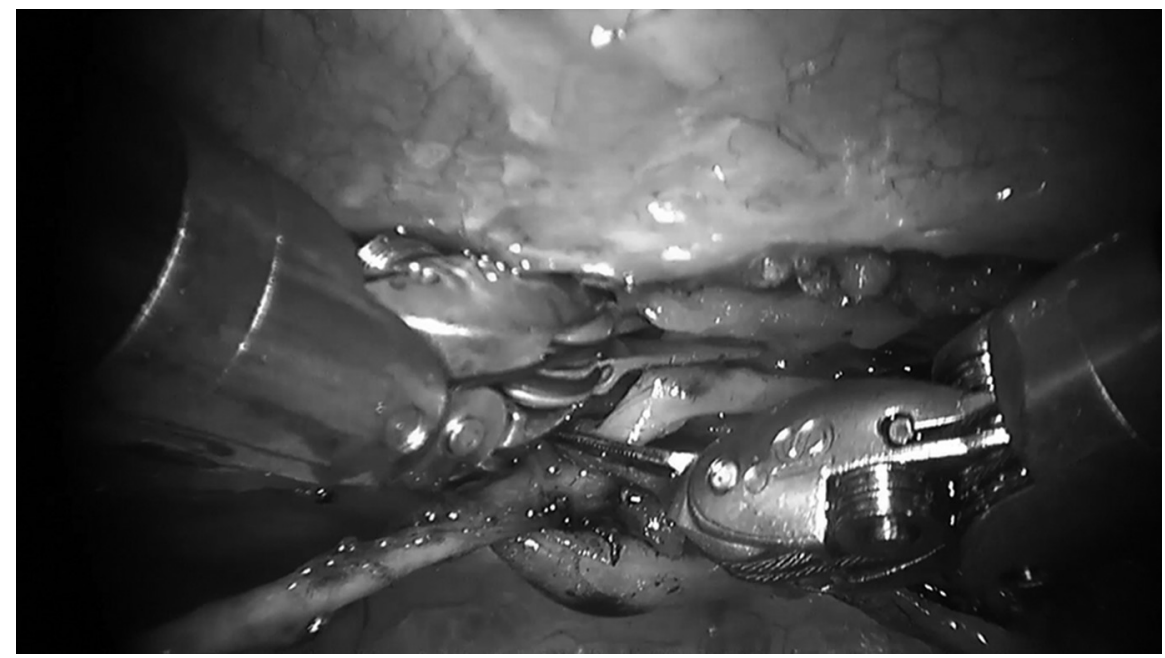

Fig. 2 Intraoperative view of robot-assisted IMA harvesting. IMA: internal mammary artery

This procedure may be an effective alternative to offpump median sternotomy. It can be safely performed and is associated with acceptable mortality and morbidity. Fast in-hospital recovery and early return to a normal lifestyle may be additional advantages of this procedure.

MIDCAB has the advantage of limited access along with the benefits of OPCAB. However, MIDCAB is limited to single-vessel revascularization because the entire length of the left IMA (LIMA) cannot be harvested by this procedure. Single-vessel MIDCAB of the LIMA to the left anterior descending (LAD) has been reported to produce excellent results with a low mortality rate. ${ }^{7)} \mathrm{A}$ surgical robot such as the da Vinci Surgical System can be used to harvest the entire length of both IMAs through only three ports. After robot-assisted bilateral IMA harvesting, MIDCAB can be performed without long incisions in the thorax. Thus, performing these procedures has a cosmetic advantage and reduces postoperative complications.

\section{TECAB}

Watanabe et al. reported the first totally endoscopic CABG on a beating heart in $1999,{ }^{8)}$ in which the left IMA was totally endoscopically harvested and anastomosed to the LAD using a customized suction endoscopic stabilizer that enabled immobilisation of the anterior wall of the left ventricle through a thoracoport in two patients. This procedure, which did not use a surgical robot, required a very advanced skill level. Stephenson et al. originally described 25 endoscopic coronary anastomoses of the coronary artery in 1998 using isolated porcine hearts in a reproduced human anatomical orientation and rib cage. ${ }^{9)}$ Loulmet et al. performed the first robotic TECAB in humans in 1998. ${ }^{10)}$ They harvested and anastomosed the left IMA to the LAD using the first-generation da Vinci surgical system in two men with cardiac arrest. Falk described the first off-pump robotic TECAB using an endoscopic stabilizing device in 2000. ${ }^{11)}$

A port access stabilizer was required to achieve TECAB, and thus an EndoWrist stabilizer was mounted on the fourth arm of the da Vinci surgical system. This suction-type endostabilizer gives the console surgeon complete control while offering better exposure and stabilization, and these have led to highly complex endoscopic procedures such as triple and quadruple CABG being performed on arrested and beating hearts.

\section{Hybrid procedure}

The indications for CABG and PCI for multivessel coronary artery disease remain controversial. ${ }^{12,13)}$ Hybrid revascularization combines minimally invasive coronary artery bypass, such as ThoraCAB and totally endoscopic CABG, with PCI; integrating these two procedures into one therapeutic modality aims to provide patients with the benefits of each successive technique in the least invasive way. The survival benefit of the left IMA for ostial or complex lesions of the LAD has been established. ${ }^{14)}$ Bonatti et al. described the feasibility and safety of simultaneous hybrid coronary revascularization. ${ }^{15,16)}$ The approach appeared safe and feasible, particularly with advances in robotic instrumentation, and it may be an evolutionary step to use a hybrid procedure that combines PCI and minimally invasive CAG. 


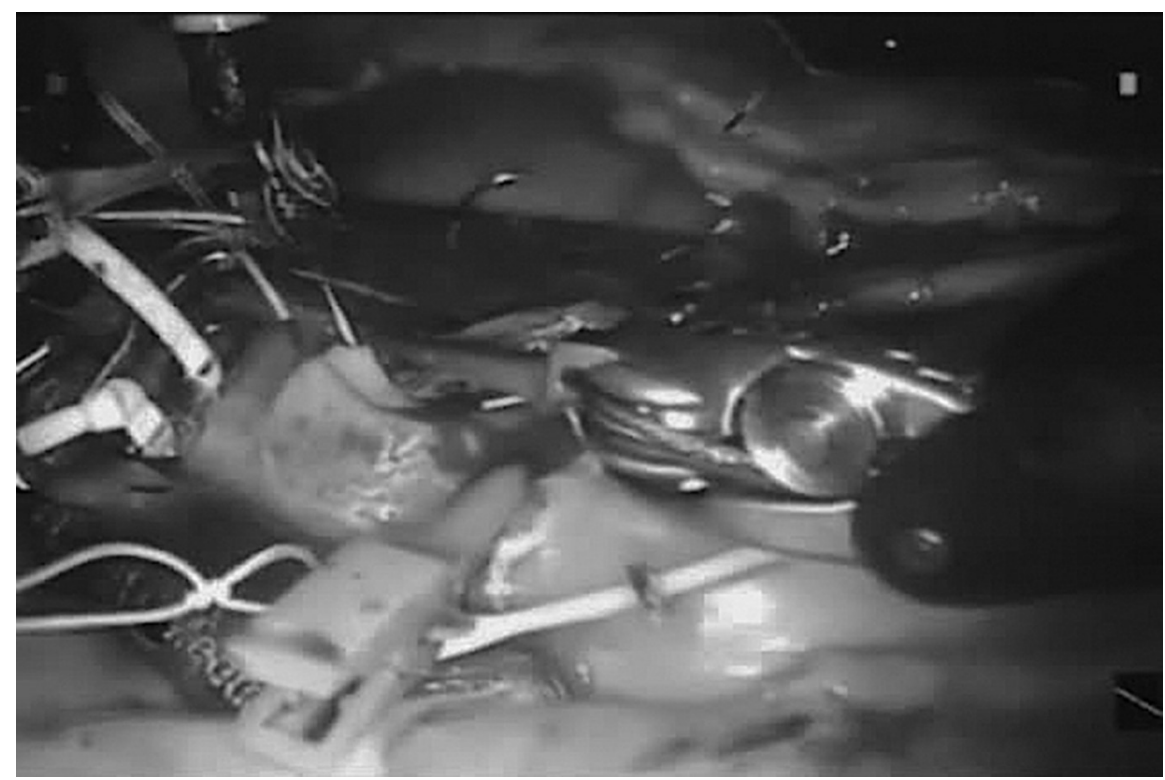

Fig. 3 Intraoperative view of totally endoscopic CABG. CABG: coronary artery bypass grafting
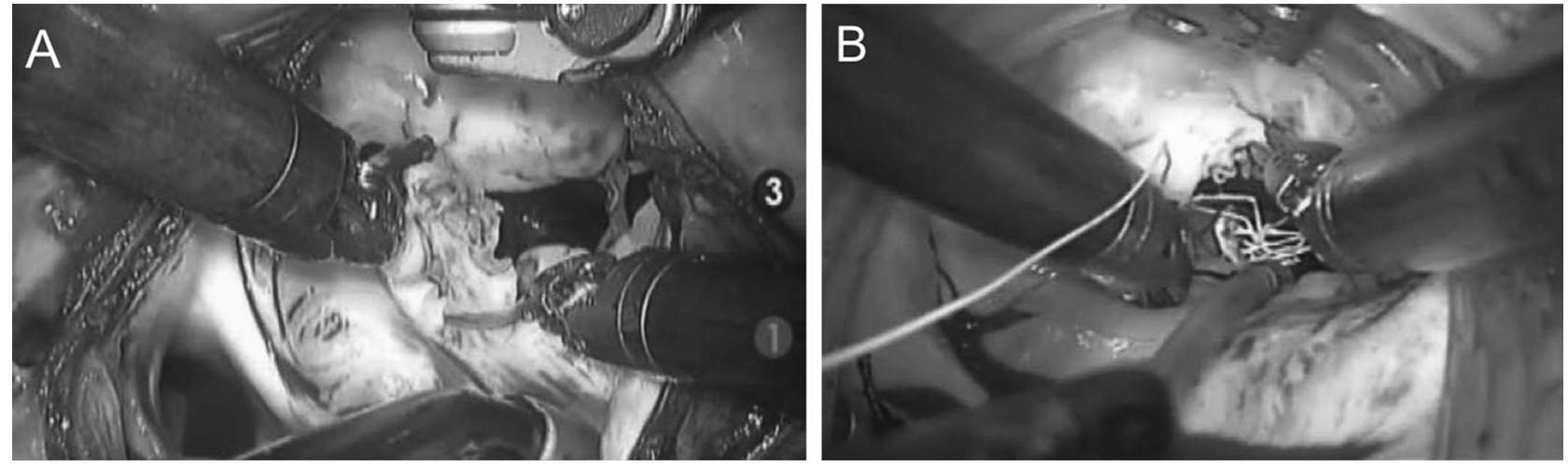

Fig. 4 Intraoperative view of robot-assisted mitral valve plasty. (A) resection and suture, (B) loop technique.

\section{Surgical technique for ischemic heart disease}

Harvesting the IMA: Patients are intubated for single-lung ventilation under general anesthesia. A robotic camera port is created in the left chest, and carbon dioxide insufflation maintains the pressure between 6 and $12 \mathrm{mmHg}$ before introducing the robotic camera. Two more instrument ports are created for robotic instruments. The mediastinum is separated from the chest wall, and the right IMA is harvested in a skeletonized fashion in a manner similar to that used in open surgery. The left IMA is then similarly dissected out (Fig. 2).

Coronary artery bypass surgery through a mini thoracotomy (ThoraCAB): After harvesting the IMA with robot assistance, a 5- to 15-cm anterolateral thoracotomy is performed on the left anterior chest. The anastomosis maneuver of the coronary artery is the same as that during the
MIDCAB procedure. The distal anastomosis is hand-sewn using a heart stabilizer, and multivessel anastomosis can be achieved during ThoraCAB. Totally endoscopic coronary artery bypass grafting (TECAB): We perform beating heart TECAB with U-clips (Medtronic, Minneapolis, MN, USA) as described by Srivastava ${ }^{17)}$ (Fig. 3). An EndoWrist stabilizer (Intuitive Surgical) is mounted on the fourth arm of the da Vinci via the fifth port placed below the xiphoid process. After securing proximal and distal control of the target coronary artery, interrupted coronary anastomosis was achieved using robotic instruments and eight small U-clips. For distal anastomosis, several methods were reported. Bonaros et al. performed a running technique using 7-0 polypropylene suture, ${ }^{18)}$ and Balkhy et al. used a coronary anastomotic connector (C-Port FREX A distal anastomotic device; Cardica, Redwood City, CA). ${ }^{19)}$ 


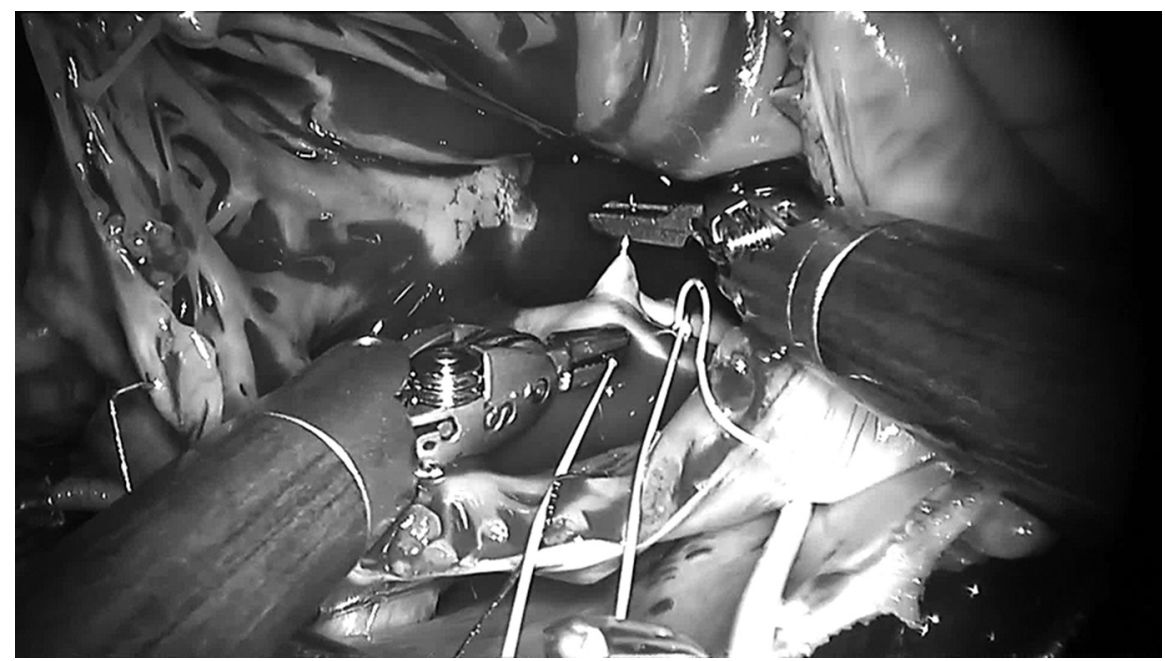

Fig. 5 Intraoperative view of robot-assisted ASD closure. ASD: atrial septal defect

\section{Robotic Surgery for Structural Heart Disease}

\section{Mitral valve surgery}

Carpentier performed the first robotic MVP using an early prototype of the da Vinci surgical system in May $1998,{ }^{20)}$ and Mohr performed the first coronary anastomosis and repaired five mitral valves using the system one week later. ${ }^{21)}$ Grossi et al. partially repaired a mitral valve using the Zeus system, ${ }^{22}$ and Chitwood performed the first complete da Vinci MVP in North America 4 days later in May 2000.23)

Two subsequent studies demonstrated that robotic mitral valve surgery is safe, the short-term results are excellent, and the mid-term durability is good. ${ }^{24,25)}$ The FDA approved the da Vinci surgical system for mitral valve surgery and atrial septal defect (ASD) repair in November 2002. Mitral valve surgery was accomplished through the same small thoracotomy incisions as the MICS procedure, since the surgical robot allowed the surgeons to complete complex maneuvers. The robotic system is now frequently applied to mitral valve surgery that customarily proceeds through a small thoracotomy, and robot-assisted totally endoscopic MVP has recently become popular. ${ }^{26)}$

Suri et al. evaluated the postoperative quality-of-life indices among asymptomatic or minimally symptomatic patients after undergoing isolated MVP for mitral valve regurgitation and concluded that those who were treated using robot-assisted MVP returned to work slightly sooner than those who were treated using a conventional transsternotomy procedure. ${ }^{27)} \mathrm{Cohn}$ et al. reported that a minimally invasive approach was associated with a reduced need for red blood cell transfusion, greater patient satisfaction, and a $20 \%$ reduction in costs compared with the conventional approach. ${ }^{28)}$

\section{Surgery for congenital heart disease}

ASD closure using direct suture repair or patches has also been accomplished with robotics. Secundum ASD or patent foramen ovale with or without mitral valve regurgitation should also be addressable with surgical robots, and sinus venosus ASDs have also been repaired in this manner. Gao et al. found that on-pump ASD repairs of the beating heart using the da Vinci surgical system without crossclamping the aorta were feasible, safe, and effective. ${ }^{29)}$

\section{Surgical technique for structural heart disease}

Patients are intubated for single-lung ventilation after general anesthesia is induced. Assisted bicaval venous drainage proceeds first like the MICS (minimally invasive cardiac surgery) procedure. The right internal jugular vein is cannulated, and the right femoral artery and vein are cannulated in the right groin. Cardiac arrest and myocardial protection are maintained using a transthoracic aortic cross-clamp. Intermittent antegrade cold blood cardioplegic solution is directly administered through the anterior chest via an angiocatheter.

Totally endoscopic mitral valve repair: A right-sided approach is taken through one 12-mm port for the robotic camera, three 8-mm ports for the robotic instruments and atrial retractor, and another 12-mm port for the delivery of sutures or the annuloplasty band. A transthoracic aortic cross-clamp is inserted through a 5-mm skin incision, and 
mitral valve plasty proceeds using standard techniques. The posterior leaflet lesion is resected and re-sutured, and the loop technique is applied to the anterior leaflet lesion using robotic instruments (Fig. 4). Annuloplasty bands are placed into the atrium with interrupted sutures for all patients.

Totally endoscopic ASD closure: The CPB setting is almost identical to that of mitral valve surgery except for the need for an additional bicaval venous clamp. The right atrium is exposed using a retraction suture and never a robotic atrial retractor. All ASD closures, such as direct closure or patch plasty with pericardium, proceed using standard techniques (Fig. 5).

\section{Disclosure Statements}

This research received no specific grant from any funding agency in the public, commercial, or not-for-profit sectors. Furthermore, none of the authors have any commercial or financial involvements in connection with this study that represent or appear to represent any conflicts of interest.

\section{References}

1) Modi P, Hassan A, Chitwood WR. Minimally invasive mitral valve surgery: a systematic review and metaanalysis. Eur J Cardiothorac Surg 2008; 34: 943-52.

2) Srivastava S, Gadasalli S, Agusala M, et al. Use of bilateral internal thoracic arteries in $\mathrm{CABG}$ through lateral thoracotomy with robotic assistance in 150 patients. Ann Thorac Surg 2006; 81: 800-6.

3) Iribarne A, Easterwood R, Chan EY, et al. The golden age of minimally invasive cardiothoracic surgery: current and future perspectives. Future Cardiol 2011; 7: 333-46.

4) Cooley DA. Con: beating-heart surgery for coronary revascularization: is it the most important development since the introduction of the heart-lung machine? Ann Thorac Surg 2000; 70: 1779-81.

5) Contini M, Lacò A, Lovino T, et al. Current results in off pump surgery. Eur J Cardiothorac Surg 1999; 16: S69-72.

6) Polomsky M, Puskas JD. Off-pump coronary artery bypass grafting - the current state. Circ J 2012; 76: 784-90.

7) Calafiore AM, Giammarco GD, Teodori G, et al. Left anterior descending coronary artery grafting via left anterior small thoracotomy without cardiopulmonary bypass. Ann Thorac Surg 1996; 61: 1658-63; discussion 1664-5.

8) Watanabe G, Takahashi M, Misaki T, et al. Beating-heart endoscopic coronary artery surgery. Lancet 1999; 354: 2131-2.

9) Stephenson ER, Sankholkar S, Ducko CT, et al. Robotically assisted microsurgery for endoscopic coronary artery bypass grafting. Ann Thorac Surg 1998; 66: 1064-7.
10) Loulmet D, Carpentier A, d'Attelis N, et al. Endoscopic coronary artery bypass grafting with the aid of computer assisted instruments. J Thorac Cardiovasc Surg 1999; 118: $4-10$

11) Falk V, Diegeler A, Walther T, et al. Total endoscopic computer enhanced coronary artery bypass grafting. Eur J Cardiothorac Surg 2000; 17: 38-45.

12) Takayama T, Hiro T, Hirayama A. Is angioplasty able to become the gold standard of treatment beyond bypass surgery for patients with multivessel coronary artery disease? Therapeutic strategies for 3-vessel coronary artery disease: OPCAB vs PCI(PCI-Side). Circ J 2010; 74: 2744-9.

13) Nishimi M, Tashiro T. Off-pump coronary artery bypass vs percutaneous coronary intervention. Therapeutic strategies for 3-vessel coronary artery disease: OPCAB vs PCI(PCI-Side). Circ J 2010; 74: 2750-7.

14) Gao C, Yang M, Wu Y, et al. Hybrid coronary revascularization by endoscopic robotic coronary artery bypass grafting on beating heart and stent placement. Ann Thorac Surg 2009; 87: 737-41.

15) Bonatti JO, Zimrin D, Lehr EJ, et al. Hybrid coronary revascularization using robotic totally endoscopic surgery: perioperative outcomes and 5-year results. Ann Thorac Surg 2012; 94: 1920-6.

16) Bonatti J, Lehr E, Vesely MR, et al. Hybrid coronary revascularization: which patients? when? how? Curr Opin Cardiol 2010; 25: 568-74.

17) Srivastava S, Gadasalli S, Agusala M, et al. Beating heart totally endoscopic coronary artery bypass. Ann Thorac Surg 2010; 89: 1873-80.

18) Bonaros N, Schachner T, Lehr E, et al. Five hundred cases of robotic totally endoscopic coronary artery bypass grafting: predictors of success and safety. Ann Thorac Surg 2013; 95: 803-12.

19) Balkhy HH, Wann LS, Krienbring D, et al. Integrating coronary anastomotic connectors and robotics toward a totally endoscopic beating heart approach: review of 120 cases. Ann Thorac Surg 2011; 92: 821-7.

20) Carpentier A, Loulmet D, Aupècle B, et al. Computer assisted open heart surgery. First case operated on with success. C R Acad Sci III 1998; 321: 437-42.

21) Mohr FW, Falk V, Diegeler A, et al. Computer-enhanced coronary artery bypass surgery. J Thorac Cardiovasc Surg 1999; 117: 1212-4.

22) Grossi EA, Lapietra A, Applebaum RM, et al. Case report of robotic instrument-enhanced mitral valve surgery. J Thorac Cardiovasc Surg 2000; 120: 1169-71.

23) Chitwood WR, Nifong LW, Elbeery JE, et al. Robotic mitral valve repair: trapezoidal resection and prosthetic annuloplasty with the da vinci surgical system. J Thorac Cardiovasc Surg 2000; 120: 1171-2.

24) Nifong LW, Chu VF, Bailey BM, et al. Robotic mitral valve repair: experience with the da Vinci system. Ann Thorac Surg 2003; 75: 438-42.

25) Nifong LW, Chitwood WR, Pappas PS, et al. Robotic mitral valve surgery: a United States multicenter trial. J Thorac Cardiovasc Surg 2005; 129: 1395-404. 
26) Rodríguez E, Kypson AP, Moten SC, et al. Robotic mitral surgery at East Carolina University: a 6 year experience. Int J Med Robot 2006; 2: 211-5.

27) Suri RM, Antiel RM, Burkhart HM, et al. Quality of life after early mitral valve repair using conventional and robotic approaches. Ann Thorac Surg 2012; 93: 761-9.
28) Cohn LH, Adams DH, Couper GS, et al. Minimally invasive cardiac valve surgery improves patient satisfaction while reducing costs of cardiac valve replacement and repair. Ann Surg 1997; 226: 421-6.

29) Gao C, Yang M, Wang G, et al. Totally endoscopic robotic atrial septal defect repair on the beating heart. Heart Surg Forum 2010; 13: E155-8. 